\title{
Single-dose BNT162b2 vaccine protects against asymptomatic SARS-CoV-2 infection
}

\author{
Michael Weekes ${ }^{1}$, Nick K Jones ${ }^{2}$, Lucy Rivett ${ }^{2}$, Chris Workman ${ }^{3}$, Mark Ferris ${ }^{3}$, Ashley \\ Shaw $^{4}$, Cambridge Covid-19 Collaboration ${ }^{5}$, Paul J Lehner ${ }^{5}$, Rob Howes ${ }^{6}$, Giles Wright ${ }^{3}$, \\ Nicholas J Matheson ${ }^{5,7}$, and Michael P Weekes ${ }^{8}$ \\ ${ }^{1}$ Affiliation not available \\ ${ }^{2}$ Clinical Microbiology \& Public Health Laboratory, Public Health England \\ ${ }^{3}$ Cambridge University Hospitals Occupational Health and Wellbeing \\ ${ }^{4}$ Cambridge University Hospitals \\ ${ }^{5}$ Cambridge Institute of Therapeutic Immunology \& Infectious Disease, University of \\ Cambridge \\ ${ }^{6}$ Cambridge COVID-19 Testing Centre \\ ${ }^{7} \mathrm{NHS}$ Blood and Transplant \\ ${ }^{8}$ Cambridge Institute for Medical Research, University of Cambridge
}

February 24, 2021

Nick K. Jones ${ }^{1,2^{*}}$, Lucy Rivett ${ }^{1,2^{*}}$, Chris Workman ${ }^{3}$, Mark Ferris $^{3}$, Ashley Shaw ${ }^{1}$, Cambridge COVID-19 Collaboration $^{1,4}$, Paul J. Lehner ${ }^{1,4}$, Rob Howes ${ }^{5}$, Giles Wright $^{3}$, Nicholas J. Matheson ${ }^{1,4,6 \mathbb{I}}$, Michael P. Weekes ${ }^{1,7 \mathbb{I}}$

${ }^{1}$ Cambridge University NHS Hospitals Foundation Trust, Cambridge, UK

${ }^{2}$ Clinical Microbiology \& Public Health Laboratory, Public Health England, Cambridge, UK

${ }^{3}$ Occupational Health and Wellbeing, Cambridge Biomedical Campus, Cambridge, UK

${ }^{4}$ Cambridge Institute of Therapeutic Immunology \& Infectious Disease, University of Cambridge, Cambridge, UK

${ }^{5}$ Cambridge COVID-19 Testing Centre and AstraZeneca, Anne Mclaren Building, Cambridge, UK

${ }^{6}$ NHS Blood and Transplant, Cambridge, UK

${ }^{7}$ Cambridge Institute for Medical Research, University of Cambridge, Cambridge, UK

*Joint first authorship

IJoint last authorship

Correspondence: mpw1001@cam.ac.uk

The UK has initiated mass COVID-19 immunisation, with healthcare workers (HCWs) given early priority because of the potential for workplace exposure and risk of onward transmission to patients. The UK's Joint Committee on Vaccination and Immunisation has recommended maximising the number of people vaccinated with first doses at the expense of early booster vaccinations, based on single dose efficacy against symptomatic COVID-19 disease. ${ }^{1-3}$ 
At the time of writing, three COVID-19 vaccines have been granted emergency use authorisation in the UK, including the BNT162b2 mRNA COVID-19 vaccine (Pfizer-BioNTech). A vital outstanding question is whether this vaccine prevents or promotes asymptomatic SARS-CoV-2 infection, rather than symptomatic COVID-19 disease, because sub-clinical infection following vaccination could continue to drive transmission. This is especially important because many UK HCWs have received this vaccine, and nosocomial COVID-19 infection has been a persistent problem.

Through the implementation of a 24 h-turnaround PCR-based comprehensive HCW screening programme at Cambridge University Hospitals NHS Foundation Trust (CUHNFT), we previously demonstrated the frequent presence of pauci- and asymptomatic infection amongst HCWs during the UK's first wave of the COVID-19 pandemic. ${ }^{4}$ Here, we evaluate the effect of first-dose BNT162b2 vaccination on test positivity rates and cycle threshold $(\mathrm{Ct})$ values in the asymptomatic arm of our programme, which now offers weekly screening to all staff.

Vaccination of HCWs at CUHNFT began on $8^{\text {th }}$ December 2020, with mass vaccination from $8^{\text {th }}$ January 2021. Here, we analyse data from the two weeks spanning $18^{\text {th }}$ to $31^{\text {st }}$ January 2021 , during which: (a) the prevalence of COVID-19 amongst HCWs remained approximately constant; and (b) we screened comparable numbers of vaccinated and unvaccinated HCWs. Over this period, 4,408 (week 1) and 4,411 (week 2) PCR tests were performed from individuals reporting well to work. We stratified HCWs $<12$ days or $>12$ days post-vaccination because this was the point at which protection against symptomatic infection began to appear in phase III clinical trial. ${ }^{2}$

26/3,252 (0.80\%) tests from unvaccinated HCWs were positive ( $\mathrm{Ct}<36)$, compared to $13 / 3,535(0 \cdot 37 \%)$ from HCWs $<12$ days post-vaccination and 4/1,989 (0.20\%) tests from HCWs [?]12 days post-vaccination $\left(\mathrm{p}=0^{*} 023\right.$ and $\mathrm{p}=0^{*} 004$, respectively; Fisher's exact test, Figure). This suggests a four-fold decrease in the risk of asymptomatic SARS-CoV-2 infection amongst HCWs [?]12 days post-vaccination, compared to unvaccinated HCWs, with an intermediate effect amongst $\mathrm{HCWs}<12$ days post-vaccination.

A marked reduction in infections was also seen when analyses were repeated with: (a) inclusion of HCWs testing positive through both the symptomatic and asymptomatic arms of the programme $(56 / 3,282(1 * 71 \%)$ unvaccinated vs 8/1,997 ( $0 * 40 \%)$ [?]12 days post-vaccination, $4 * 3$-fold reduction, $\left.\mathrm{p}=0^{*} 00001\right)$; (b) inclusion of PCR tests which were positive at the limit of detection ( $\mathrm{Ct}>36,42 / 3,268(1 * 29 \%)$ vs $15 / 2,000(0 * 75 \%)$, $1 * 7$-fold reduction, $\mathrm{p}=0^{*} 075$ ); and (c) extension of the period of analysis to include six weeks from December $28^{\text {th }}$ to February $7^{\text {th }} 2021\left(113 / 14,083\left(0^{*} 80 \%\right)\right.$ vs $5 / 4,872\left(0^{*} 10 \%\right), 7^{*} 8$-fold reduction, $\left.\mathrm{p}=1 \times 10^{-9}\right)$. In addition, the median $\mathrm{Ct}$ value of positive tests showed a non-significant trend towards increase between unvaccinated HCWs and HCWs $>12$ days post-vaccination ( $23 * 3$ to $30 * 3$, Figure), suggesting that samples from vaccinated individuals had lower viral loads.

We therefore provide real-world evidence for a high level of protection against asymptomatic SARS-CoV-2 infection after a single dose of BNT162b2 vaccine, at a time of predominant transmission of the UK COVID19 variant of concern 202012/01 (lineage B.1.1.7), and amongst a population with a relatively low frequency of prior infection ( $7.2 \%$ antibody positive). ${ }^{5}$

This work was funded by a Wellcome Senior Clinical Research Fellowship to MPW (108070/Z/15/Z), a Wellcome Principal Research Fellowship to PJL (210688/Z/18/Z), and an MRC Clinician Scientist Fellowship (MR/P008801/1) and NHSBT workpackage (WPA15-02) to NJM. Funding was also received from Addenbrooke's Charitable Trust and the Cambridge Biomedical Research Centre. We also acknowledge contributions from all staff at CUHNFT Occupational Health and Wellbeing and the Cambridge COVID-19 Testing Centre. 


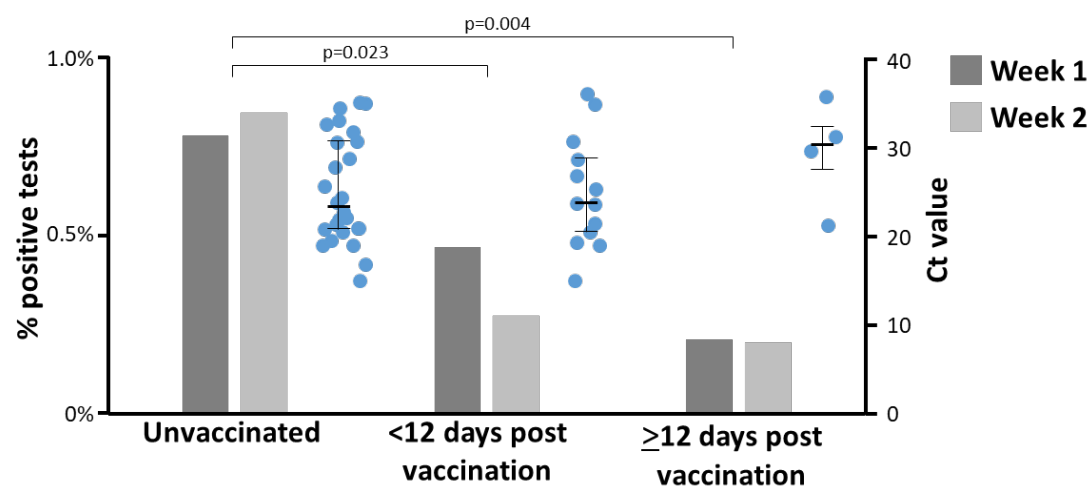

Figure : Proportion of HCWs testing positive for SARS-CoV-2 in the CUHNHFT asymptomatic screening programme (grey bars; week 1, 18/01/2021-24/01/2021; week 2, 25/01/2021-31/01/2021) and Ct values of positive tests $(\mathrm{CT}<36$; blue dots; both weeks). RT-PCR targeting the SARS-CoV-2 ORF1ab genes was conducted at the Cambridge COVID-19 Testing Centre (part of the UK Lighthouse Labs Network). For proportions testing positive, $\mathrm{p}$ values for pair-wise comparisons of unvaccinated HCWs with HCWs $<12$ days or $>12$ days post-vaccination are shown (Fisher's exact test; both weeks). For Ct values, medians \pm interquartile ranges are shown.

\section{References}

1. Department of Health and Social Care. Optimising the COVID-19 vaccination programme for maximum short-term impacthttps: //www. gov. uk/government/publications/prioritising-the-firstcovid-19-vaccine-dose-jcvi-statement/optimising-the-covid-19-vaccination-programme-formaximum-short-term-impact

2. Polack FP, Thomas SJ, Kitchin N, et al. Safety and Efficacy of the BNT162b2 mRNA Covid-19 Vaccine. $N$ Engl J Med2020;383(27):2603-15. DOI: 10.1056/NEJMoa2034577

3. Voysey M, Clemens SAC, Madhi SA, et al. Safety and efficacy of the ChAdOx1 nCoV-19 vaccine (AZD1222) against SARS-CoV-2: an interim analysis of four randomised controlled trials in Brazil, South Africa, and the UK. Lancet 2021;397(10269):99-111. DOI:https://doi.org/10. 1016/S0140$6736(20) 32661-1$

4. Rivett L, Sridhar S, Sparkes D, et al. Screening of healthcare workers for SARS-CoV-2 highlights the role of asymptomatic carriage in COVID-19 transmission. eLlife 2020;9. DOI: 10.7554/eLife.58728

5. Cooper DJ, Lear S, Watson L, et al. A prospective study of risk factors associated with seroprevalence of SARS-CoV-2 antibodies in healthcare workers at a large UK teaching hospital. medRxiv 2020. DOI: https://doi.org/10.1101/2020.11.03.20220699

\section{Cambridge COVID-19 Collaboration}

Amy Amory, Steve Baker, Emma Bateman, Aklima Begum, Moushima Begum, John Bradley, Michael Brennan, Helen Burn, Caroline Crofts, Sharon Dawson, Gordon Dougan, Renny Feather, Louise Free, Katie Friel, Claire Gildea, Ian Goodfellow, Lizz Grimwade, Ravi Gupta, Susan Hall, Sophie Hannan, James Hayes, Aleksandra Hosaja, Lidia James, Jill Jardin, Nathalie Kingston, Paul A. Lyons, Patrick H. Maxwell, Sue Mott, Sarah Mugavin, Joyce Mwiya, Ravi Prakash Nallattil, Willem H. Ouwehand, Elle Page, Marina Perez, Natalie Quinnell, Caroline Saunders, Kenneth G.C. Smith, Maria Stafford, Charlotte Summers, Despiona Tatsi, James E.D. Thaventhiran, Sharon Thomas Johnson, Mark Toshner, Lesley Turner, Kate Wall, Ben Warne, Karis Watson, Jo Wright 